\title{
The Current Funding Landscape of Bevacizumab (Avastin) As Front-Line Treatment for Advanced Ovarian Cancer: A Health Economics Dilemma
}

\author{
Shiru Liu* \\ Doctor of Medicine and Master of Surgery, Fellow of Royal College of Physicians and Surgeons of Canada, Canada \\ *Corresponding author: Shiru Liu, Doctor of Medicine and Master of Surgery, Fellow of Royal College of Physicians and Surgeons \\ of Canada \\ To Cite This Article: Shiru Liu, The Current Funding Landscape of Bevacizumab (Avastin) As Front-Line Treatment for Advanced Ovarian Can- \\ cer: A Health Economics Dilemma. Am J Biomed Sci \& Res. 2021 - 12(3). AJBSR.MS.ID.001752. DOI: 10.34297/AJBSR.2021.12.001752.
}

Received: 㘹 January 19, 2021; Published: March 31, 2021

Key words: Gynecologic Malignancies, Ovarian Cancer, Monoclonal Antibody, High-Risk Features, Pharmaceutical, Jurisdictions, Intravenous, \$2,500 CAD Per Injection, Drug, Chemotherapy

\section{Introduction}

Ovarian cancer is the most common cause of death in women with gynecologic malignancies [1]. Until recently, standard treatment in the front-line setting consists of combination chemotherapy with debulking surgery [2]. Despite optimal upfront therapy, the estimated 5 -year overall survival is approximately $40 \%$ and prognosis is much worse for those with stage IV disease or other high-risk features [3]. Systemic therapy for ovarian has been evolving in the past decade with the emergence of targeted therapy, such as Avastin (bevacizumab), a monoclonal antibody targeting vascular endothelial growth factor, currently manufactured by the pharmaceutical company Hoffman-La Roche. It has shown promising activity and survival benefit in several large randomized clinical trials of women with high-risk advanced ovarian cancer $[4,5]$, leading to FDA approval of this drug in the front-line setting and representing a new standard treatment option for this disease. However, cost-effectiveness analyses have shown conflicting results, with many studies suggesting this may not be a costeffective intervention, particularly in jurisdictions such as Canada, where a public health care system is in place [6-10].

In Canada, after Roche submitted a funding request through Health Canada in 2015, the pan Canadian Oncology Drug Review (pCODR) expert review committee (pERC) only recommended conditional funding of Avastin in subgroup high risk population, providing an improvement in its cost-effectiveness to an acceptable level [11]. Following these recommendations, provincial jurisdictions negotiated the price of the drug with Roche to then provide their own policies individually regarding funding of this expensive intravenous therapy, currently estimated to cost approximately $\$ 2,500$ CAD per injection, excluding the cost of administration such as chair time, nursing, and staff personnel. In addition, many jurisdictions have already approved the funding of this drug in the recurrent setting, although patients must not have received this in the front-line setting. As such, many oncologists may choose not to prescribe this in the first line setting and wait for disease recurrence, which occurs in most patients. Furthermore, providers who prescribe the drug differ in training background. While many are medical oncologists who are internal medicine trained, a large proportion of prescribers of chemotherapy for gynecologic malignancies in Canada and the United States are gynecology oncologists, who are surgically trained.

As such, there are inherent differences in the choice of drug prescription, with medical oncologists more inclined and comfortable to prescribe new targeted therapies. Gynecology oncologists have been reluctant to adopt Avastin in their practice mainly due to fear of bowel perforation, a complication that can occur with this drug in patients undergoing debulking surgery. Finally, where public funding is not available, patients with private drug insurance have been able to obtain adequate co-payment for their treatment when deemed appropriate. This ultimately leads to inequity in access to front-line therapy for ovarian cancer and an 
inherent heterogeneity in oncologic outcome based on geography and provider. Challenges of cancer drug therapy in health policies: the suppliers

To better understand the reasoning behind the funding polices that are made, it is important to consider the incentives and objectives of the different stakeholders involved. Pharmaceutical companies have become the largest markets in the field of oncology, especially with the modern era of targeted therapy as we move towards personalized medicine. According to the Canadian Centre for Health Economics, one must consider the incentives for the producers, consumers, and regulators of drugs to develop good pharmaceutical policy [12]. The goal of the producers, i.e., the pharmaceutical companies, is to maximize profit. They are given incentives to provide a stream of new drug therapies while also restricting their costs. The competition that exists amongst pharmaceutical companies allows for lower drug prices and increased choices for the consumers, which ultimately benefits society at large. Fundamentally, the goal of such policies is to maximize social benefit. The consumers, on the other hand, seek to obtain individual health as a capital good, or health capital. In the world of oncology, good health is measured by survival time and the quality of this time.

As such, for most randomized phase 3 clinical trials involving a new drug therapy, the primary endpoints required to obtain regulatory approval are usually overall survival and/or quality of life [13]. With improvement in drug therapy over time, it has become increasingly difficult to achieve these endpoints, because researchers must constantly find a treatment that is better than the current standard of care, which continues to evolve with time [14]. Many trials now use endpoints such as Progression-Free Survival (PFS) as a surrogate marker for overall survival and have been able to obtain accelerated regulatory approval based on preliminary results [15]. However, many phases 2 trials with promising PFS results ultimately do not show overall survival benefit in phase 3 setting due to various factors, so it is important avoid non-validated surrogate endpoints in registration trials [14]. In addition, quality of life in these studies have become increasingly important to report and have become a standard measure of effectiveness in drug therapy.

The cost of research and development of a new cancer drug has become increasingly expensive [16]. The cost of production must also consider cost of failures, acknowledging high failure rate and few truly effective drugs, with only $50 \%$ of the phase 2 studies that progress onto phase 3 [17]. Investigators must rely heavily on pharmaceutical companies to achieve such large-scale multi-centre clinical trials, as it is extremely difficulty to conduct such studies with publicly supported funds such as university or government grants. The investigators, many of whom are oncologists with research background, have the incentive to improve the health outcome of cancer patients. As such, there may be, at times, an inherent misalignment in objectives between the different suppliers, as profit seeking behaviour does not always equate to better patient care. For oncology drugs there is often a monopoly on the supply side, with a drug that is solely manufactured through one pharmaceutical company until it either goes off patent, acquires a biosimilar, or develops a "me-too" version by other companies [18]. Government from the public sector must provide its input, as with the pCODR system, to grant access to these drugs. Therefore, the pharmaceutical sector remains contentious as the role of the government grows over time in determining pricing of drugs.

Finally, probably the most important factor in determining access to new oncotherapy is the availability of private drug insurance. This is especially the case for health jurisdictions such as the United States, where most cancer therapies are reimbursed at least partially by insurance plans. In Canada, in places where bevacizumab was not approved initially in ovarian cancer, patients with private drug insurance who are eligible for additional bevacizumab therapy were able to obtain coverage for this treatment through their insurance. Because it was not a publicly covered medication, patients had to receive it through a private infusion clinic as well, which usually requires out-of-pocket cost. Patients with comprehensive drug coverage are more likely to be offered and receive new cancer therapies [19-21]. And, presumably, those with such drug coverage have higher sociodemographic profile, with higher health literacy and higher income. This concept of income inequality leading to health disparity is not unique to bevacizumab in ovarian cancer, nor is it unique to cancer care. Ultimately, the social determinants of health go beyond drugs insurance and explore complex social factors that influence population health [22].

\section{A World on Biosimilars}

Biosimilars are compounds that are molecularly similar, but not identical, to an existing licensed and approved biologic in the market. They are intended to treat the same condition but at a lower cost. They also require a highly rigorous evaluation through clinical trials to establish their efficacy and safety [23]. Comparative and pharmacokinetics studies must be performed before they can be approved and licensed. Of note, these are different from generics. Currently licensed and approved biosimilar of bevacizumab include ABP 215/MVASI (developed by Amgen), BCD-021 (Biocad), BI 695502 (Boehringer Ingelheim), and PF-06439535 (Pfizer) [24]. In Canada, MVASI is currently approved for the treatment of metastatic colorectal cancer and non-small cell lung cancer [25]. These products have been shown to be cost-effective and highly similar in efficacy when compared to bevacizumab and have resulted in improved access to biologic therapy [26]. In the future, the medical 
community anticipate that bevacizumab biosimilar may play a larger role in gynecologic malignancies as well, including ovarian cancer. From a cost perspective, this would certainly yield improved cost-effectiveness and potentially, improved access to therapy.

\section{A Few Discussions Point}

Worldwide, bevacizumab is only available as a standard treatment in developed countries, such as Canada and the United States, western European countries, and Australia. It is also in these countries where the landmark clinical trials took place. As such, fundamentally, there is geographical disparity associated with bevacizumab access and availability. Within those countries and jurisdictions, differences public and private funding policies, prescribing trend, and institutional experience further result in unequal in access to drug therapy, ultimately leading to disparity in cancer outcomes. From a health economics perspective, however, the policies in place and the processes involved leading to the current reality may not be as disconsolate as it seems. To benefit society at large and to improve population health in a sustainable way, the focus has always been on optimal allocation of scarce resources within a budget constraint [27]. Allotting more resources to bevacizumab for ovarian cancer would mean less resources to another disease or another tumor site. This is especially important to consider in the context of the cost-effectiveness analyses, given the available data only revealed a statistically significant survival benefit in a subgroup population with high-risk features, and even then, the benefit is measured in months.

In the world of oncology, a few months' difference in survival can certainly be deemed clinically relevant. However, without overall survival benefit in the whole population, can progression-free survival really be enough for society to decide to pay for a drug? Can quality of life be the sole primary endpoint in cancer patients? What is the threshold for additional number of months in life expectancy to be truly meaningful? The moral dilemma that regulatory bodies must face in these situations can be challenging. What ultimately is deemed effective or beneficial seems more difficult to determine than one would expect. A one size fits all approach cannot be applied given the heterogeneity of the diseases, but some uniform resource rationing system must exist to tackle opportunity costs. In the case of ovarian cancer and bevacizumab, the economical solution may very well be to find the optimal biomarker that allows us to identify the subset of patients who would respond best to targeted therapy, employing a biosimilar when possible.

The solution to regulating private insurance remains a challenging one. While it may be tempting to wish for universal health coverage so that everyone has access to the same products, it is also the competition resulting in unequal access that allows pharmaceutical companies to produce newer and better treatments which then become more accessible to everyone. It may be counterintuitive, and there is certainly a fine balance, but a small degree of inequality in health is sometimes required for longterm improvement in health. Private cancer centers in the United States are one example of a sector that is extremely competitive but also highly efficient in research and development of cancer therapy. Coming back to the supplier issue, we need and depend on large competitive pharmaceutical companies to provide a constant supply of various drug therapies to ultimately obtain competitive pricing and better treatments.

\section{Concluding remarks}

In summary, access to bevacizumab for front-line treatment of ovarian cancer remains challenging due to various reasons including public funding policies, private insurance coverage, physician and institutional factors, and concerns regarding its costeffectiveness. In Canada and in the United States, most jurisdictions have access to bevacizumab, but implementation of this regimen is not well captured. Biosimilars have emerged as a cheaper alternative and have yet to capture the gynecology landscape. Much more work is needed to improve the outcomes of ovarian cancer patients, but theories within health economics may shed light to better understand objectives, incentives, and behaviors of the different stakeholders involved in cancer care.

\section{Conflict of Interest}

The author reports no financial disclosures.

\section{References}

1. Siegel RL, Miller KD, Jemal A (2018) Cancer statistics, 2018. CA: Cancer J Clinicians 68(1): 7-30.

2. Morgan RJ, Armstrong DK, Alvarez RD, Jamie NB, Kian B, et al. (2016) Ovarian Cancer, Version 1.2016, NCCN Clinical Practice Guidelines in Oncology. J Natl Compr Canc Netw 14(9): 1134-1163.

3. Jayson GC, Kohn EC, Kitchener HC, Ledermann JA (2014) Ovarian cancer. Lancet 384(9951): 1376-1388.

4. Perren TJ, Swart AM, Pfisterer J, Jonathan AL, Eric PL, et al. (2011) A phase 3 trial of bevacizumab in ovarian cancer. N Engl J Med. 365(26): 2484-2496.

5. Burger RA, Brady MF, Bookman MA, Gini Fleming, Bradley JM, et al. (2011) Incorporation of bevacizumab in the primary treatment of ovarian cancer. N Engl J Med 365(26): 2473-2483.

6. Cohn DE, Kim KH, Resnick KE, O’Malley DM, Straughn JM Jr, (2011) At What Cost Does a Potential Survival Advantage of Bevacizumab Make Sense for the Primary Treatment of Ovarian Cancer? A Cost-Effectiveness Analysis. J Clin Oncol 29(10): 1247-1251.

7. Mehta DA, Hay JW, (2014) Cost-effectiveness of adding bevacizumab to first line therapy for patients with advanced ovarian cancer. Gynecol Oncol 132(3): 677-683.

8. Juliet EW, Jiaru Bai, Lindsey EM, Robin K, Ramez NE, et al. (2018) Cost-effectiveness of maintenance therapy in advanced ovarian cancer: Paclitaxel, bevacizumab, niraparib, rucaparib, olaparib, and pembrolizumab. J Clin Oncol 36(15): 5508-5508. 
9. Hinde S, Epstein D, Cook A, Embleton A, Perren T, Sculpher M, (2016) The Cost-Effectiveness of Bevacizumab in Advanced Ovarian Cancer Using Evidence from the ICON7 Trial. Value Health 19(4): 431-439.

10. Duong M, Wright E, Yin L, Martin NI, Ghatage P, et al. (2016) The costeffectiveness of bevacizumab for the treatment of advanced ovarian cancer in Canada. Current Oncology 23(5): 461-467.

11. Pcodr_bevacizumab_avastin_oc-fn_rec. pp1-11.

12. Laporte A, Ferguson BS (2015) Changing the Way We Think About Drug Prices: Insights from Economics. October.

13. Administration FAD, (2018) Clinical Trial Endpoints for the Approval of Cancer Drugs and Biologics. December 2018: 1-19.

14. Tannock IF, Amir E, Booth CM, Saroj Niraula, Alberto O, et al. (2016) Relevance of randomised controlled trials in oncology. Lancet Oncol 17(12): 560-567.

15. Zhang J, Pilar MR, Wang X, Jingxia Liu, Herbert P, et al. (2020) Endpoint surrogacy in oncology Phase 3 randomised controlled trials. Br J Cancer 123(3): 333-334

16. Meropol NJ, Kevin AS, (2007) Cost of cancer care: issues and implications. J Clin Oncol 25(2): 180-186.

17. Arrowsmith J, (2011) Phase III and submission failures: 2007-2010. Nature Reviews Drug Discovery 10(2): 87.

18. (2012) Pills Patents \& Pro ts II. pp1-50.

19. Abdelsattar ZM, Hendren S, Wong SL, (2017) The impact of health insurance on cancer care in disadvantaged communities. Cancer 123(7): 1219-1227.
20. Temkin SM, Rimel BJ, Bruegl AS, Gunderson CC, Beavis AL, et al. (2018) A contemporary framework of health equity applied to gynecologic cancer care: A Society of Gynecologic Oncology evidenced-based review. Gynecol Oncol 149(1): 70-77.

21. Graham S, Hallisey E, Wilt G, Flanagan B, Rodriguez JL, et al. (2019) Sociodemographic disparities in access to ovarian cancer treatment. Ann Cancer Epidemiol 3: 10.

22. Marmot M, Friel S, Bell R, Houweling TAJ, Commission on Social Determinants of Health, et al. (2008) Closing the gap in a generation: health equity through action on the social determinants of health. Lancet 372(9650): 1661-1669.

23. Lemery SJ, Ricci MS, Keegan P, McKee AE, Pazdur R, (2017) FDA's Approach to Regulating Biosimilars. Clin Cancer Res 23(8): 1882-1885.

24. Monk BJ, Huh WK, Rosenberg JA, Jacobs I, (2017) Will bevacizumab biosimilars impact the value of systemic therapy in gynecologic cancers? Gynecol Oncol Res Pract 4(1): 7.

25. CADTH Biosimilar Summary Dossier. pp1-53.

26. Chopra R, Lopes G, (2017) Improving Access to Cancer Treatments: The Role of Biosimilars. J Glob Oncol 3(5): 596-610.

27. Folland S, Goodman AC, Stano M, (2007) The Economics of Health and Health Care. Prentice Hall. 